\title{
La responsabilidad social como factor determinante en el prestigio y reconocimiento de las empresas
}

\author{
Social responsibility as a determining factor in the prestige and \\ recognition of companies
}

1 Halder Yandry Loor Zambrano Universidad Técnica de Manabí, Portoviejo, Ecuador halder.loor@utm.edu.ec

2 Martha Lissette Zambrano Moreira Universidad Estatal de Bolívar, Guanujo, Ecuador mzambrano@ueb.edu.ec

3 Danilo Renato Valle Arellano.

Universidad Estatal de Bolívar, Guanujo, Ecuador dvalle@ueb.edu.ec

\author{
(iD) https://orcid.org/0000-0001-9828-9209 \\ https://orcid.org/0000-0003-2633-5071
}

Artículo de Investigación Científica y Tecnológica Enviado: 06/12/2021

Revisado: 21/12/2021

Aceptado: 28/01/2022

Publicado:05/02/2022

DOI: https://doi.org/10.33262/ap.v4i1.1.153

Loor Zambrano, H. Y., Zambrano Moreira, M. L., \& Valle Arellano, D. R. (2022). La responsabilidad social como factor determinante en el prestigio y reconocimiento de las Cítese: $\quad$ empresas . AlfaPublicaciones, 4(1.1), 176-187. https://doi.org/10.33262/ap.v4i1.1.153

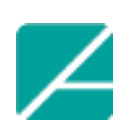

Ciencia Digital
ALFA PUBLICACIONES, es una Revista Multidisciplinar, Trimestral, que se publicará en soporte electrónico tiene como misión contribuir a la formación de profesionales competentes con visión humanística y crítica que sean capaces de exponer sus resultados investigativos y científicos en la misma medida que se promueva mediante su intervención cambios positivos en la sociedad. https://alfapublicaciones.com

La revista es editada por la Editorial Ciencia Digital (Editorial de prestigio registrada en la Cámara Ecuatoriana de Libro con No de Afiliación 663) www.celibro.org.ec

Esta revista está protegida bajo una licencia Creative Commons Attribution Non Commercial No Derivatives 4.0 International. Copia de la licencia: http://creativecommons.org/licenses/by-nc-nd/4.0/ 


\section{Palabras}

claves:

RSE, prestigio, reconocimiento, competitividad, rentabilidad

\section{Keywords:}

CSR, prestige, recognition, competitiveness, profitability

\section{Resumen}

El objetivo de esta investigación se basa en evaluar la responsabilidad social como factor determinante en el prestigio y el reconocimiento de las empresas. La metodología aplicada se basa en un diseño bibliográfico de tipo documental. El buen prestigio y el reconocimiento de las empresas frente al mercado y la sociedad se debe a diversos factores, y uno de ellos es la responsabilidad social, donde se ve reflejada las buenas intenciones y las acciones éticas, honestas, transparentes, y sinceras que tienen las empresas tanto con el medio ambiente, sus trabajadores y la sociedad y por medio de estas lograr cumplir objetivos trazados que les ayude a alcanzar beneficios y mayor competitividad y rentabilidad. Como conclusión se puede mencionar que la responsabilidad social empresarial es mucho más que solo ejercer acciones filantrópicas, son procesos complejos, que requieren de habilidades y buenas estrategias empresariales para lograr cumplir los objetivos planteados. Esto permite que la empresa y todos los que conforman su estructura comprendan la importancia de ser socialmente responsables, no solamente en beneficio de la empresa sino también de forma personal profesional y beneficiando a las comunidades que le rodean, además permite que las empresas reduzcan sus costos, lo que puede generar una mejor calidad de vida tanto para la empresa como para los colaboradores.

\section{Abstract}

The objective of this research is based on evaluating social responsibility as a determining factor in the prestige and recognition of companies. The applied methodology is based on a bibliographic design of a documentary type. The good prestige and recognition of companies in the market and society is due to various factors, and one of them is social responsibility, where good intentions and ethical, honest, transparent and sincere actions are reflected. companies both with the environment, their workers and society and through these achieve goals that help them achieve benefits and greater competitiveness and profitability. In conclusion, it can be mentioned that corporate social responsibility is much more than just exercising philanthropic actions, they are complex processes that require skills and good business strategies to achieve the objectives set. This allows the company and all those 


\begin{abstract}
who make up its structure to understand the importance of being socially responsible, not only for the benefit of the company but also in a personal professional way and benefiting the surrounding communities, it also allows companies to reduce their costs., which can generate a better quality of life for both the company and employees.
\end{abstract}

\title{
Introducción
}

El prestigio según la Real Academia Española (RAE, 2014) es la estima pública de alguien o algo, y que es producto de su mérito. Etimológicamente hablando el termino prestigio posee diversos significados y uno de ellos, se refiere a cuando una persona posee influencia o autoridad, o un truco que se usa para engañar (Anders, 2022).

También se puede mencionar que esta palabra proviene del latín praestigium y se formaba por el prefijo prae (delante) y el verbo stringere que significa apretar con fuerza, y esta hace referencia a la ilusión que generaba un mago con sus trucos a los espectadores del show. Posteriormente en Francia pasado el siglo XVIII, se utilizó la palabra "prestige" cuyo significado era renombre, influencia y así llego a la lengua española.

En la actualidad, su significado no ha tenido un cambio considerable en referencia al adquirido por los franceses, y es usado en muchos aspectos de la sociedad, a nivel político, económico y social. En este sentido a nivel empresarial ha sido de gran relevancia obtener un prestigio de su marca y sus productos, bienes o servicios frente a sus clientes y sus trabajadores, por tanto, han tratado de lograr avances y mejoras a nivel laboral y productivo que les permite generar reconocimiento en su círculo económico.

Una buena imagen, un trato correcto y respetuoso a sus empleados, buenos niveles de productividad, una buena recepción de la marca a sus clientes, así como un buen trato al ambiente y la sociedad son aspectos que generan prestigio a las empresas que apliquen estos principios y este reconocimiento no solo es interno (sus trabajadores) sino también externo (sociedad y empresas).

De la misma manera Ayala (2021), menciona que es importante demostrar las acciones positivas que realizan las empresas pues esta situación mostrará el valor que tiene la organización, y al mismo tiempo aumentara su reputación a su alrededor. De igual manera, indica que en situaciones críticas económicas muchas organizaciones prefieren recortar presupuesto de publicidad y comunidad, y esto es un error pues a pesar de que genere un gasto debe ser visto como una inversión a futuro, pues esto dará frutos al generar buena comunicación y demostrar buenas acciones. 
Asa mismo Ayala (2021), menciona una serie de pautas fundamentales para lograr una buena reputación organizacional y se mencionan a continuación:

- Más allá de las acciones positivas propias del negocio para fortalecer la imagen, también importa y en gran medida, la transparencia, el comportamiento corporativo, la ética, la ayuda a la sociedad, la responsabilidad social, el trato a los colaboradores, el respeto a la ley, entre otros, que conforman un todo de la organización.

- Cada nivel de las empresas es importante para consolidar la reputación de la organización, pues eso demuestra que sus empleados y cualquier nivel jerárquico poseen ética, valor, transparencia y otros valores importantes y positivos que demuestran conocimiento y experiencia.

- Cualquier empresa, sea grande o pequeña debe tener claro que la comunicación corporativa es fundamental y es una manera de hacer negocios, es importante mostrar atención e interés con sus trabajadores, sus gremios, sus "stakeholders" pues esta estrategia sirve para construir reputación.

- Más allá de comunicación corporativa todas las personas deben mantener el mismo enfoque, y el mismo mensaje para que pueda llegar a quien se desee la información de forma correcta y esto trae consigo que todos se conviertan en voceros y que no solo el presidente ejecutivo sea quien transmita lo que la empresa quiere demostrar (Ayala, 2021).

Actualmente la sociedad más allá del aspecto financiero, tiene gran interés en el apoyo que las empresas prestan hacia el medio ambiente y a las comunidades, por tanto, la reputación y el reconocimiento que una empresa pueda mostrar va de la mano de las acciones que esta ejecute en beneficio de lo antes mencionado, pues estas impactan de forma positiva o negativa en la apreciación que la sociedad pueda tener de ellas (Herrera et al., 2016).

En este sentido, es evidente que las empresas deben ser más conscientes de trabajar en la responsabilidad social para demostrar sus buenos intereses, así como la capacidad que tienen para comprometerse en mejorar situaciones que pueden ser controladas desde la empresa en beneficio de la sociedad, el ambiente y sus trabajadores.

El reconocimiento empresarial también es muy importante para lograr prestigio frente a la competencia, una marca bien posicionada ayuda a alcanzar reconocimiento en el mercado. Pero más allá de reconocer la marca de una empresa, esta desea lograr que la empresa sea reconocida por las buenas acciones que esta realiza a la sociedad y en apoyo a sus trabajadores, de manera que sea un precedente para sus iguales.

De esta manera Rivera (2017), menciona que existe una serie de responsabilidades éticas que las empresas deben cumplir para sus trabajadores y la comunidad y estas son: 
- Servir a la sociedad con productos o servicios que sean útiles y adecuados, considerando condiciones justas.

Los consumidores son influenciados por la manera en que la empresa muestre su producto y que este a su vez sea amigable con el ambiente y cubra las necesidades de la población, de manera que más allá de vender el producto este satisfaga dentro de las posibilidades.

- Respetar los derechos humanos de los trabajadores, demostrando buenas condiciones de trabajo, donde la seguridad, su desarrollo profesional y su salud laboral sea favorecido.

- Respetar el medio ambiente, evitando en gran medida contaminarlo, esto se logra minimizando la generación de residuos y racionalizando el uso de los recursos naturales.

El planeta viene sufriendo grandes cambios climáticos, producto de la contaminación y el uso inadecuado de sus recursos que han generado fenómenos naturales, por tanto, las empresas conscientes de esta situación deben mejorar el uso de sus recursos naturales y energéticos para crear conciencia ambiental y ayudar a preservar el ambiente Fuente especificada no válida.

- Seguimiento de la gestión de los recursos y residuos: es necesario que la empresa tenga un uso consciente de sus recursos para evitar pérdidas.

- Revisión de la eficiencia energética de la empresa.

- Implicar a los empleados en las buenas prácticas de la responsabilidad social: las buenas acciones que hace la empresa deben ser transmitidas a sus empleados para que puedan ser partícipes de ellas, lo que ayuda a crear conciencia y valores en ellos.

- Mejorar las posibilidades y oportunidades de la comunidad donde se establece la empresa: esto se logra cuando las empresas brindan oportunidades de empleo a la comunidad que le rodea, mejorando la calidad de vida de estos. Asimismo, puede brindar capacitación sobre algún tema para que las personas puedan desarrollar actividades económicas que le beneficien (Rivera, 2017).

De esta manera el objetivo de esta investigación se basa en evaluar la responsabilidad social como factor determinante en el prestigio y el reconocimiento de las empresas. La metodología aplicada se basa en un diseño bibliográfico de tipo documental.

\section{Metodología}

Esta investigación fue desarrollada a través del diseño bibliográfico, el cual según Palella \& Martins (2010), un diseño bibliográfico se basa en una revisión rigurosa y profunda de material documental. Donde se realizan diversos análisis y se establece la relación de 
entre dos o más variables. En este sentido se realizó la búsqueda de documentos de carácter científico técnico para obtener resultados coherentes.

De igual manera, se menciona que la investigación es de tipo documental el cual es un proceso de búsqueda que se realiza en diversas fuentes para lograr obtener la información deseada, y la vez analizarla, ordenarla y describir las ideas más importantes plasmadas en los documentos seleccionados.

En este sentido Palella \& Martins (2010), mencionan que, las investigaciones de tipo documental se concretan exclusivamente en la recopilación de información en diversas fuentes, e indaga sobre temas que otros han escrito que se analizan para obtener resultados propios.

\section{Resultados y Discusión}

De manera general, la responsabilidad social es un compromiso que adquieren los miembros de una sociedad de forma individual o grupal y que repercute en ellos, y que puede ser positiva o negativa (Jácome-Lara et al., 2017).

De igual manera se indica que existen diferentes tipos de RS, entre estas:

- Responsabilidad ambiental.

- Responsabilidad política

- Responsabilidad moral y ética.

- Responsabilidad social empresarial

Se profundiza en esta investigación la responsabilidad social empresarial, que está basada en todas las obligaciones a nivel legal y ético que tienen las organizaciones con la comunidad y la sociedad en general, más allá de solo obtener ventajas a nivel empresarial, existen tres valores diferentes: valores ambientales, sociales y económicos (Jácome-Lara et al., 2017).

La RSE es definida por García (2014), citada por Remache-Rubio et al. (2018) como "la capacidad de valorar las consecuencias que tienen en la sociedad las acciones y decisiones que toman las diferentes personas y organizaciones como parte del logro de sus propios objetivos y metas" (p.552). De esta manera, es como las empresas deben participar de forma activa en el compromiso social.

En este sentido Jácome-Lara et al. (2017), muestran una serie de modelos relacionado con los valores antes mencionados y se observan en la tabla 1. 


\section{Tabla 1}

Modelos ligados a los valores de la RSE

\begin{tabular}{cl}
\hline Modelo & \multicolumn{1}{c}{ Concepto } \\
\hline & $\begin{array}{l}\text { Las empresas comprometidas con este modelo, tratan de utilizar siempre } \\
\text { materia prima no contaminante y material de reciclaje, intentado no } \\
\text { solamente cumplir estrictamente las leyes y normativas en materia de }\end{array}$ \\
Modelo de protección & $\begin{array}{l}\text { contaminación y medio ambiente, sino que tratan de ir un paso por delante, } \\
\text { del medio ambiente }\end{array}$ \\
& $\begin{array}{l}\text { poniendo en marcha programas de recuperación de parajes naturales o de } \\
\text { conservación de flora y fauna. }\end{array}$
\end{tabular}

Las empresas que optan por este modelo centran sus esfuerzos en mejorar las condiciones laborales, económicas y sociales de las zonas donde ponen

Modelo de fomento de valores éticos y sociales

Modelo centrado en unos valores económicos justos y equilibrados en marcha sus plantas de producción. Por otro lado, procuran establecer unos códigos morales y de conducta entre todos los miembros de la empresa con el objeto de asegurar unas condiciones laborales justas y un entorno laboral positivo.

Es desarrollado por aquellas empresas, que tratan de conseguir unas relaciones más lógicas y justas entre todos los eslabones de la cadena del mercado: productores, proveedores y clientes, buscando el equilibrio entre los recursos empleados y la repartición de beneficios

Fuente: Jácome-Lara et al. (2017)

La responsabilidad social empresarial según menciona Perdiguero (2003, citado por Calderón, 2007) es:

La Responsabilidad Social Empresarial es un término que se ha utilizado desde la década de los sesenta pero que ha cobrado especial fuerza a partir de 1990 cuando se idealiza a la empresa como una institución que, además de tener responsabilidad y obligaciones legítimas frente a sus accionistas, sería también responsable de los efectos que generan sus actividades en la sociedad y el impacto que generaría en el ambiente. (p.246)

Sin embargo, muchas empresas se han tomado a la ligera la RSE alejándose de los principios con los que fue creada originalmente, pues utilizan sus "buenas acciones" para hacer mercadotecnia, para mostrar una imagen fuerte hacia sus consumidores, sin haber realizado algún cambio significativo. Estas acciones traen consigo que la empresa llegue a ser reconocida como una empresa con falta de ética, valores institucionales y su reputación se ve afectada por ello.

Por este tipo de situaciones, existen diversas organizaciones e instituciones internacionales que se encargan de brindarles asesoría a las empresas, así como también guiarles a través de propuestas, y fomentar en ellos la transparencia al momento de aplicar la RSE (Remache-Rubio et al., 2018). Algunas de estas instituciones son: 
- La comisión Económica para América Latina y el Caribe (CEPAL)

- El Instituto Ethos de Responsabilidad Social

- International Organization for Standardization (ISO).

Esta última (ISO) creó un documento que sirve de guía para que las empresas tengan ideas más claras al momento de aplicar la RSE y este promueve el entendimiento común de la RSE, así según Remache-Rubio et al. (2018) son:

- Fomenta que las empresas realicen actividades que vayan mas allá de solo cumplir lo que la ley les exige.

- Definir los principios y prácticas relativos a la responsabilidad social.

- Presenta las materias y asuntos fundamentales relacionados con la responsabilidad social con un toque holístico

- Promueve la comunicación de los compromisos y el desempeño relacionados con la RS.

- Promueve el aumento de la toma de conciencia y creación de competencias en responsabilidad social.

- Recomienda como mejorar la credibilidad en materia de responsabilidad social

- Recomienda como mejorar el desempeño

- Es compatible con otros sistemas de gestión.

\section{La responsabilidad social y sus beneficios}

En vista de todos los alcances que puede tener el aplicar correctamente la Responsabilidad social es importante demostrar cuáles son sus beneficios y entre estos se encuentran:

Beneficios sociales: ser socialmente responsables como empresa es fundamental para lograr estos beneficios, esto se demuestra en las buenas prácticas empresariales, en el uso adecuado de los recursos, y en la correcta eliminación de los desechos, así como también los programas de orientación y preparación a su personal para que se apropien de estos valores que les permitirá alcanzar ventajas competitivas a nivel regional, nacional e internacional (Castro, 2017).

Beneficios Económicos: el éxito económico de una empresa radica en su rentabilidad, sin embargo, las empresas al mostrar que son socialmente responsables requieren invertir parte de su rentabilidad en esos proyectos sociales, que tal vez en el momento es un gasto, pero finalmente es una inversión pue esto les permite lograr apoyo de entes gubernamentales, de otras empresas e instituciones que le permitirán mover fuerzas y lograr mas apoyo a la sociedad.

Beneficios ambientales: cuando las empresas son responsables con el ambiente mas allá de solo cumplir con las leyes, surgen una serie de beneficios directos que les permite 
lograr reconocimiento y prestigio en la sociedad (Castro, 2017). En este sentido se menciona que estas acciones de sensibilización ambiental que generan mayor competitividad y confianza entre la empresa y la comunidad permiten:

- Reducción de los costos ambientales y costos generales de las empresas

- Permite acceder a mercados más exigentes y restringidos por razones ambientales diferenciándose con respecto a sus competidores,

- Mejora la imagen general de la empresa y credibilidad frente a sus clientes.

- Permite introducir mejoras técnicas y funcionamiento en la propia empresa, facilitando la actividad empresarial, acceso a ciertos contratos para reducir las enfermedades y accidentes laborales con la implantación de nuevas tecnologías.

\section{Algunas razones por la cual una empresa debe ser socialmente responsable}

Lograr esto es bastante complejo, pero no imposible para las organizaciones sin embargo algo importante que deben entender las empresas es la habilidad que deben tener para lograrlo, de manera que no quede en el intento y puedan ser realmente responsables, donde surja una interacción social que traiga consigo beneficios para todas las partes (Martín, 2016).

En este sentido las principales razones para que una empresa sea socialmente responsable, según Martín (2016), son:

- Favorece la confianza, el sentido de pertenencia de los empleados, lo cual permite que aumente la disposición hacia el trabajo, que disminuya la rotación de personal y por consiguiente los costos de reclutamiento;

- Beneficia la calidad y con ella la satisfacción de clientes. Además, se mejora la imagen de la empresa, lo que contribuye a mantener la clientela y el acceso a nuevos mercados;

- Incrementa el apoyo de la comunidad y de autoridades locales, lo cual facilita el flujo de procedimientos y la seguridad;

- Reduce, recicla y reutilizan muchos recursos, permitiéndose así el ahorro de grandes sumas, la recuperación de inversiones y la optimización de recursos.

De igual forma existen otras razones que reflejan la importancia de que las organizaciones sean conscientes del alcance que tiene la responsabilidad social empresarial y se muestran en la tabla 2. 
Tabla 2

Razones para que las empresas sean socialmente responsables

\begin{tabular}{cl}
\hline Razones & \multicolumn{1}{c}{ Concepto } \\
\hline & Se favorece la imagen positiva que tienen los consumidores y \\
Fidelización de los clientes & clientes. Lo cual puede renovar el compromiso de los mismos.
\end{tabular}

Mejoran la imagen de empresa ante la comunidad empresarial y de los inversionistas, las empresas líderes son destacadas en los

Atracción de inversionistas

Mejora la relación con el entorno e incrementa la influencia de la empresa en la sociedad

Reduce los costos operativos

Permite acceder e influir en líderes de opinión medios de comunicación, incluidos en fondos éticos $\mathrm{y}$ recomendadas a los fondos de inversión.

Publicar información honesta y precisa, emprender acciones socialmente comprometidas hace que se mejore la imagen de la empresa frente a la sociedad civil y genera nexos que pueden desencadenar en proyectos sociales conjuntos.

Las acciones de RSE focalizadas especialmente en reducir la contaminación del medio ambiente pueden reducir gastos y la improductividad.

Una empresa que destine parte de su presupuesto en acciones sociales o ambientales y no esconda información será destacada en los medios de comunicación y permita influir positivamente en los líderes de opinión.

Fuente: Martín (2016)

\section{Conclusiones}

- La responsabilidad social empresarial es mucho más que solo ejercer acciones filantrópicas, son procesos complejos, que requieren de habilidades y buenas estrategias empresariales para lograr cumplir los objetivos planteados.

- Esto permite que la empresa y todos los que conforman su estructura comprendan la importancia de ser socialmente responsables, no solamente en beneficio de la empresa sino también de forma personal profesional y beneficiando a las comunidades que le rodean.

- Además, al aplicar la responsabilidad social empresarial de forma honesta y con transparencia esto genera prestigio que a la vez permite que la sociedad, el mercado económico, la comunidad y los entes gubernamentales reconozcan las organizaciones por las buenas acciones que realizan.

La RSE permite que las empresas reduzcan sus costos, lo que puede generar una mejor calidad de vida tanto para la empresa como para los colaboradores. 


\section{Referencias Bibliográficas}

Anders, V. (2022). Etimología de prestigio.

Ayala, S. (2021). El valor del prestigio empresarial.

Calderón, D. (2007). Responsabilidad social empresarial. Marco de exigibilidad a las empresas en la correcta observancia y respeto a los derechos humanos. Entretextos 9(27), 241-249.

Castro, A. (2017). La responsabilidad social empresarial y la competitividad en las organizaciones empresariales. Revista INNOVA ITFIP 1(1), 78-89.

Herrera, J., Larrán, M., Lechuga, M., \& Martínez, D. (2016). Responsabilidad social en las pymes: análisis exploratorio de factores explicativos. Revista de ContabilidadSpanish Accounting Rewiew 19(1), 31-44.

Jácome-Lara, I., Salazar-Corrales, A., \& Borja-Brazales, Y. (2017). La responsabilidad social empresarial en la gestión administrativa. Dominio de las Ciencias 3(3), 1147-1158.

Martín, M. (09 de marzo de 2016). Responsabilidad social empresarial.

Palella, S., \& Martins, F. (2010). Metodología de la investigación cuantitativa. Caracas, Venezuela: FEDUPEL, Fondo Editorial de la Universidad Pedagógica Experimental Libertador.

Real Academia Española, RAE. (2014). Definición de prestigio.

Remache-Rubio, M., Villacis-Torres, S., \& Guayta-Topanta, N. (2018). La responsabilidad social empresarial vista desde un enfoque teórico. Dominio de las Ciencias 4(1), 550-568.

Rivera, S. (2017). Branding. Estrategia integral de gestión empresarial. Arequipa, Perú: Texto Universitario. 
El artículo que se publica es de exclusiva responsabilidad de los autores y no necesariamente reflejan el pensamiento de la Revista Alfa Publicaciones.

\section{Ciencia}

El artículo queda en propiedad de la revista y, por tanto, su publicación parcial y/o total en otro medio tiene que ser autorizado por el director de la Revista Alfa Publicaciones.
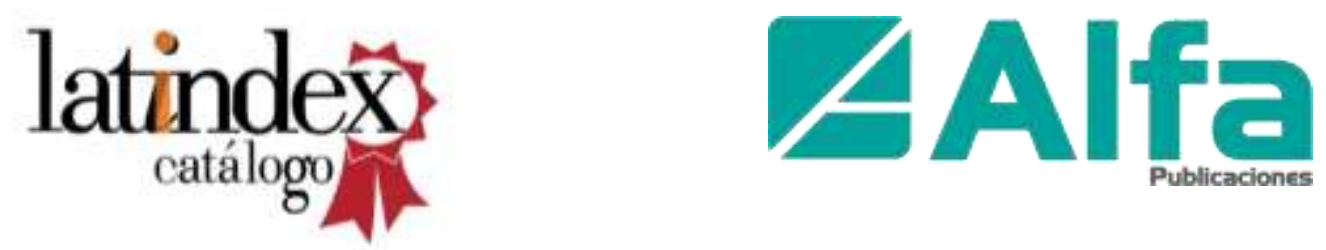

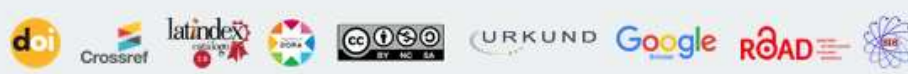
DLatinREV

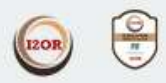

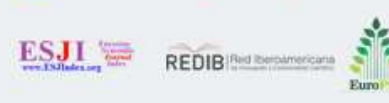

$\underbrace{}_{\text {wizdom.ai }} \mathrm{O}_{\text {OpenAIRE }}^{+}$

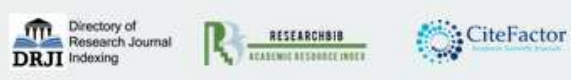

